convert the short waves into radiation able to penetrate the gelatin.

Having had occasion recently to photograph spectra of low intensity in this region, we were surprised to find that better results could be obtained with ordinary plates than with plates recommended as having much higher ultra-violet sensitivity. We have therefore carried out some experiments with the view of clearing up this apparently anomalous behaviour.

The procedure was as follows. A heavy-current discharge tube, through which passed a slow stream of air, provided a steady source of the $\gamma$-bands of nitric oxide. These bands are characterised by strong, well-defined heads, with long branches slowly degraded in the direction of shorter wave-lengths. The range of intensity thus available makes this band system very suitable for comparison of plate characteristics in the ultra-violet. Using a Hilger E2 quartz spectrograph, identical sets of exposures in logarithmic steps were made on each plate under test. Ordinary plates (Imperial) were compared in this way with Schumann plates (Hilger and Agfa), special ultra-violet plates (Eastman and Agfa), $Q$-plates (Ilford), and Imperial plates treated with such common sensitisers as anthracene, sodium salicylate, vaseline and various mineral oils.

In most cases, especially when the source is weak, the spectroscopist wishes

(a) to obtain a measurable record of the spectrum with the least possible exposure, and

(b) to reproduce, as a range of perceptible density differences, the intensity differences present in the actual spectrum.

Our results show that

$\left(a^{\prime}\right)$ every plate tested, except the Ilford $Q 2$ plate, gives less blackening for short exposures than does an ordinary plate. That is to say, an ordinary plate fulfils condition $(a)$ better than the 'sensitised' plates, with this one exception.

$\left(b^{\prime}\right)$ the 'sensitised' plates are capable of showing more intensity contrast than ordinary plates, because they have a greater saturation density. This increased density, however, is obtained only by prolonging exposure beyond that which is needed to reach saturation with ordinary plates.

If, therefore, it is desired merely to detect a weak spectrum in this region, it is undesirable to use 'sensitised' plates. If, on the other hand, the source of radiation is intense, or short exposure time is not aimed at, these plates will give greater contrast and higher saturation density than ordinary ones.

It is perhaps also worth noting that considerable over-exposure in this region does not produce on an ordinary plate densities comparable with those attainable in the nearer ultra-violet or visible region. Apart altogether from considerations of fogging, excessive exposure is detrimental in that it smooths out what little intensity contrast is present in a correctly exposed plate. This is shown by the fact that, on an over-exposed plate, even such a spectrum as that of NO appears almost continuous.

A full account of the experiments will be published shortly.

Royal College of Science.

A. Hunter.

South Kensington,

London, S.W.7.

June 3.

${ }^{1}$ For example, Hopfield and Appleyard, J. Opt. Soc. America, 22, 488 (1932). Weichmann, $Z$. wiss. Phot, 34, 140 (1935).

\section{Nova Lacertæ, 1936}

SLIT spectra of the nova have been obtained at this Observatory on every night, with one exception, since the discovery was announced on June 19.

The most prominent feature in the spectra is the broad emission bands of the Balmer series of hydrogen, $H \alpha$ being particularly bright. The width of the $H \beta$ and $H \gamma$ emissions on the evening of June 22 was about 50 angstroms. These emissions are flanked on the side of shorter wave-length by rather weak absorption lines. The more displaced component of the two $H \gamma$ absorptions indicated a velocity of about $-1100 \mathrm{~km}$./sec. on the morning of June 20 ; this rose to about $-1900 \mathrm{~km}$. $/ \mathrm{sec}$. by the evening of June 22 .

Fe II, Ti II, Mg II, and Ca II are also represented by broad weak emission bands and displaced absorp. tion lines. These absorption lines are weak and hazy, but the sharp interstellar $H$ and $K$ lines are strongly shown on a plate taken on the evening of June 22.

The general appearance of the spectrum is in sharp contrast with that of Nova Herculis, 1934, at a corresponding stage of development.

\section{T. W. WORMELL.}

J. C. DoBbiE.

Solar Physics Observatory, C'ambridge. June 26.

\section{The Background of the Galaxies}

IN NATure of May 30, M. Leontovski ${ }^{1}$ shows that "To a most sensitive eye, the background of the galaxies would appear as a dark red." Since the irresolvable background consists of nebulæ receding with nearly the speed of light, the age of these nebulæ, as observed, reckoned in our own time. scale, must be approximately one half the present age of our own surroundings; that is, if $t$ is the con. ventional value of the age of the universe, $\frac{1}{2} t$ is the age of the observed background ${ }^{2}$. Combining these results, we see that the background realizes the poet's dream of

$$
\text { "A rose-red city, half as old as time.", }
$$

19 Northmoor Road,

E. A. Mrlne. Oxford.

1 NATURE, 137, 904 (1936)

2 Mon. Not. Roy. Ast. Soc., 93, 674 (1935) ; "Relativity, Gravitation and World-Structure" (1935), p. 108 (diagram).

${ }^{3}$ J. W. Burgon, "Petra", Newdigate Prize Poem, 1815.

\section{Glycosides of Madder}

The glycoside obtained from various species of Galium and Rubia already described in NATURE ${ }^{\mathbb{t}}$ has now been found to be a primveroside of purpurin carboxylic acid.

The sugar of ruberythric acid, the glycoside of alizarin, which has been known for many years, has been isolated in the pure crystalline condition from the products of enzymic hydrolysis and identified as primverose. A primveroside of rubiadin has also been obtained from the roots of Galium Verum.

The three glycosides are rapidly hydrolysed by enzymes present in members of the Primulaceæ. They would appear to be the first examples known of primverosides occurring in the Rubiaceæ.

R. HrLL.

Biochemical Laboratory,

D. RrChter.

Cambridge. June 2.

1 Nature, 134, 628 (1934). 